\title{
Cytotoxic T Cell Response and Conserved Viral Epitopes: Considerations for Vaccination Against Newly Emerging Pandemic Influenza Strains
}

By Travis W. Marfleet

The current influenza vaccination strategy aims at the induction of a neutralizing antibody response against viral surface proteins from specific strains expected to have an increased prevalence in the approaching flu season. Selective pressure against these viral surface proteins such as hemagglutinin (HA) and neuraminidase (NA) drives viral antigenic drift, resulting in new seasonal variants. This vaccination approach requires the production of yearly formulations to target emerging antigenically drifted seasonal influenza strains ineffectively controlled by neutralizing antibody developed against previous seasonal strains.

Following the recent influenza A (H1N1) pandemic, it has been shown that a safe, effective vaccine may be developed and distributed following the emergence of a novel pandemic strain. Although the H1N1 pandemic had global health and economic consequences, the impact in terms of mortality was far below global mortality rates of seasonal influenza ${ }^{1-3}$, and was marginal compared with past influenza pandemics ${ }^{4,5}$. The limited pathogenicity of H1N1 (2009), in addition to effective epidemiological surveillance allowed for production and distribution of a safe and effective vaccine based on the current vaccine production strategy. Although this unique strategy is currently the best option, it is flawed because a novel strain must be circulating within the population prior to production of a vaccine against the new strain. Upon emergence of a highly virulent influenza strain, the time between strain emergence and vaccine distribution allows for viral spread, and leads to significant mortality rates. Hence, there is a need for implementation of a new vaccination strategy effective against previously unrecognized influenza strains. Although there is merit to mass vaccine stockpiling efforts undertaken against predicted pandemic strains, such as avian $\mathrm{H} 5 \mathrm{~N} 1$, they are

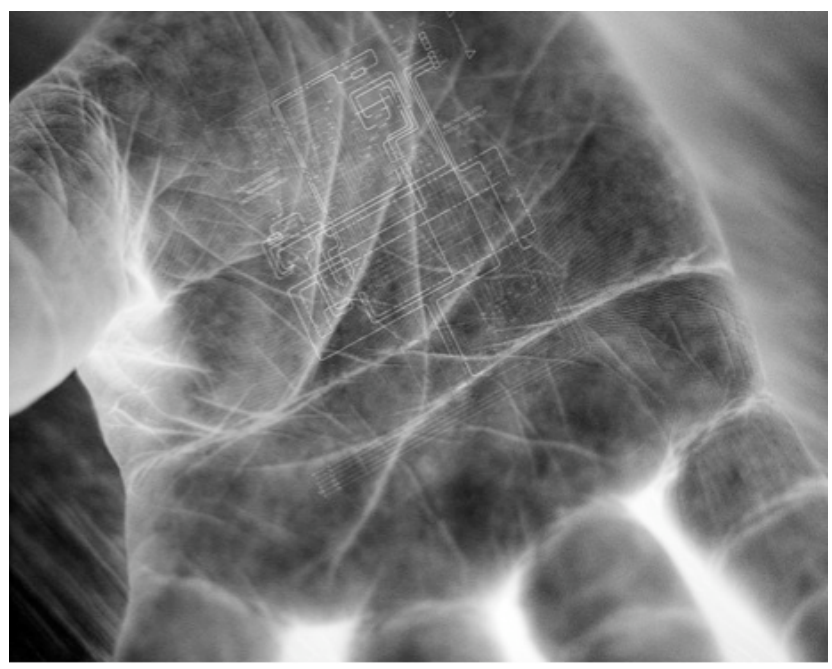

directed against a single strain and therefore unable to protect against the possible emergence of numerous pandemic viral variants over time.

The development of a universal vaccination platform to provide a broad, protective (heterosubtypic) immune response is needed to protect against newly emerging pandemics. Thus, an attractive vaccination strategy would be directed towards the development of a robust cytotoxic $\mathrm{T}$ lymphocyte (CTL) response against the influenza epitopes highly conserved across viral strains, such as the internal nucleoprotein (NP) and matrix protein 1 (M1) ${ }^{6,7}$. Both proteins contribute to the internal viral structure but do not effectively elicit a neutralizing antibody response, which is attempted by current seasonal vaccinations and would be sacrificed for the ability to generate a protective heterosubtypic host response.

The development of a robust CTL response to viral antigens depends upon the crosspresentation of viral peptides by the major histocompatibility complex I (MHC-I), which loads the viral peptides in the cytoplasm of infected cells. Thus, the antigen must be delivered to the cytoplasm 
of host cells which can be achieved by various methods, such as DNA vaccine constructs, live attenuated influenza viruses (LAIV), or recombinant viral vectors expressing influenza proteins. Split subunit or inactivated vaccines are not ideal in this case as they primarily elicit an antibody response. Live attenuated or cold-adapted vaccines access the cytoplasmic compartment with greater efficiency and elicit a stronger CTL response. Numerous studies have attempted to deliver native antigen or peptide representing conserved viral epitopes, via DNA constructs or recombinant viruses, to generate a broadly protective response in animal models with varying success (see Reference 8 for review). Several studies have demonstrated a protective response to multiple viral subtypes characterized by a strong cellular immune response following viral challenge in animal models ${ }^{9-11}$, in addition to characterizing the human CTL response to influenza vaccination (see Reference 12 and 13 for review).

Although an effective vaccine was developed following emergence of the 2009 H1N1 pandemic strain, our current vaccination strategy will likely fail following the emergence of a highly transmissible and virulent pandemic influenza virus. The development of vaccines aimed at generating a stable and lasting CTL response to protect against a broad range of seasonal and previously unrecognized influenza strains is the ideal approach to prevent a global pandemic. DNA vaccines or recombinant viruses expressing such epitopes represent a possible method of antigen delivery to the host-cell cytoplasm. Recent research has led to numerous possibilities for the development of effective vaccine vectors or antigen delivery systems that will facilitate a heterosubtypic immune response to emerging pandemic influenza.

\section{Acknowledgements}

I would like to thank Dr. C Havele and Chris Rudulier from the Department of Microbiology \& Immunology for their valuable feedback during the preparation of this article.

\section{References}

1. Seasonal Influenza: The Disease. Centers for Disease Control and Prevention; 2009 [cited $2010 \mathrm{March}$ ]; Available from: http://www.cdc.gov/flu/about/disease/index.htm.
2. Fact Sheet no. 211 - Influenza (seasonal). World Health Organization; 2009 [cited 2010 March]; Available from: http://www.who.int/mediacentre/factsheets/fs211/en/.

3. Pandemic (H1N1) 2009 - update 56. World Health Organization; 2009 [cited 2010 March]; Available from: http://www.who.int/csr/don/2009_07_01a/en/index.html.

4. Swedish KA, Conenello G, Factor SH. First Season of 2009 H1N1 Influenza. Mt Sinai J Med. 2010 Jan 1;77(1):103-13.

5. Hilleman M. Realities and enigmas of human viral influenza: pathogenesis, epidemiology and control. Vaccine. 2002;20(25-26):3068-87.

6. Effros RB, Doherty PC, Gerhard W, Bennink J. Generation of Both Cross-Reactive and Virus-Specific T-Cell Populations after Immunization with Serologically Distinct Influenza-a Viruses. Journal of Experimental Medicine. 1977;145(3):557-68.

7. Zweerink HJ, Courtneidge SA, Skehel JJ, Crumpton MJ, Askonas BA. Cytotoxic T-Cells Kill Influenza-Virus Infected-Cells but Do Not Distinguish between Serologically Distinct Type-a Viruses. Nature. 1977;267(5609):354-6.

8. Stambas J, Guillonneau C, Kedzierska K, Mintern JD, Doherty PC, La Gruta NL. Killer T cells in influenza. Pharmacology and Therapeutics. 2008 Nov 1;120(2):186-96.

9. Adar Y, Singer Y, Levi R, Tzehoval E, Perk S, Banet-Noach $\mathrm{C}$, et al. A universal epitope-based influenza vaccine and its efficacy against H5N1. Vaccine. 2009 Jan 1;27(15):2099107.

10. Chen Q, Kuang H, Wang H, Fang F, Yang Z, Zhang Z, et al. Comparing the ability of a series of viral protein-expressing plasmid DNAs to protect against H5N1 influenza virus. Virus Genes. 2009 Feb 1;38(1):30-8.

11. Price GE, Soboleski MR, Lo C-Y, Misplon JA, Pappas C, Houser $\mathrm{KV}$, et al. Vaccination focusing immunity on conserved antigens protects mice and ferrets against virulent H1N1 and H5N1 influenza A viruses. Vaccine. 2009 Nov 5;27(47):6512-21.

12. Rimmelzwaan GF, McElhaney JE. Correlates of protection: novel generations of influenza vaccines. Vaccine. 2008 Sep 12;26 Suppl 4:D41-4.

13. Rimmelzwaan GF, Fouchier RAM, Osterhaus ADME. Influenza virus-specific cytotoxic $\mathrm{T}$ lymphocytes: a correlate of protection and a basis for vaccine development. Curr Opin Biotechnol. 2007 Dec 1;18(6):529-36.

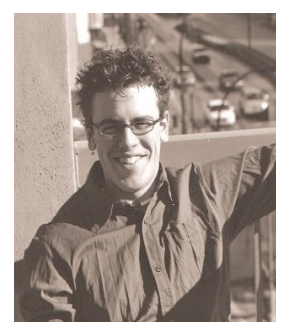

Author Profile

Travis Marfleet is currently pursuing a MSc degree in Microbiology \& Immunology at the University of Saskatchewan. His research interest is infectious diseases with focus on the host immune response to viral infections. He is mainly interested in immune class regulation and its role in host defense against pathogens. 